\title{
TO STUDY THE ROLE OF YOGA INTERVENTION ON PHYSIOLOGICAL PARAMETERS IN PEOPLE SUFFERING FROM DIABETES MELLITUS
}

\author{
Dr.Anil Kumar \\ Medical Consultant, AHS, Medical Director, AMD, Shree Sai Hospital and Trauma Centre, \\ Rewa Road Saraiya Mujaffapur, Bihar
}

Article DOI: https://doi.org/10.36713/epra7916 DOI No: 10.36713/epra7916

\begin{abstract}
Type 2 diabetes (DM2) has become a leading public health issue globally, with estimated 366 million people affected in 2011 [1]. This figure represents a more than twofold rise in the last three decades and parallels the growing pandemic of obesity and the increasingly widespread adoption of Western lifestyles [1, 2]. Worldwide prevalence is expected to continue rising in both industrialized and developing countries [ $\underline{2}, \underline{3}]$, with numbers projected to reach 552 million adults by 2030 [ㄴ, $\underline{4}]$. DM2 is now a leading cause of death and disability and significantly increases risk for both macrovascular complications, such as atherosclerosis, and microvascular complications, such as retinitis, diabetic neuropathy, and renal disease [ $\underline{5}]$.

The study also tried to find out the relationship between QoL and yoga intervention among type 2 diabetes patients. Hence, a quantitative approach was considered to be appropriate and adopted for the study. The quality of life of working population with Type 2 Diabetes mellitus with yoga intervention was observed in this study. it was indicated that the quality of life of the working population suffering from diabetes mellitus with inclusion of yoga in their daily routine has proven beneficial for their health as a result of their significantly decreased glucose levels..
\end{abstract}

KEY WORDS: Quality of life, Diabetes mellitus, yoga.

\section{INTRODUCTION}

Apart from its spiritual philosophy, yoga has been utilized as a therapeutic tool to achieve positive health and cure disease. This concept is promoted in Hath yoga and Ghatasthayoga by the yoga preceptors. Interest has been evinced in this direction by many workers and studies on the effect of yoga on hypertension, diabetes, asthma, obesity and other common ailments have been carried out.

DM2 is typified by hyperglycemia in the presence of insulin resistance [6]. Other key related hemodynamic and metabolic abnormalities characterizing DM2 include elevated blood pressure, dyslipidemia, and chronic inflammation, as well as hypercoagulation and increased oxidative stress $[\underline{5}, \underline{10}-$
12]. Risk for DM2 rises with increasing age and is further elevated in certain racial and ethnic groups, including non-Hispanic blacks, Asians, Native Americans, and Pacific Islanders [13, 14]. However, while race, age, genetic predisposition, and other nonmodifiable factors are important in the pathogenesis of DM2, lifestyle factors, particularly, physical inactivity, overnutrition, and related obesity, are thought to be primarily responsible for the current global diabetes epidemic [1, 15]. Other contributing lifestyle-related factors include chronic stress, impaired sleep, and smoking $[\underline{2}, \underline{10}, \underline{16}, \underline{17]}$. In fact, lifestyle factors may account for $90 \%$ of incident diabetes cases [2] and are significant predictors of DM2-related complications and mortality [18]. Thus, a central 


\section{SJIF Impact Factor 2021: 8.013| ISI I.F.Value:1.241| Journal DOI: 10.36713/epra2016 ISSN: 2455-7838(Online) EPRA International Journal of Research and Development (IJRD) Volume: 6 | Issue: 7 | July 2021 - Peer Reviewed Journal}

element of DM2 care is lifestyle management, which is considered critical to the prevention of acute complications and the reduction of risk for long term complications []].

\section{INCIDENCE}

Type 2 diabetes (DM2) has become a leading public health issue globally, with estimated 366 million people affected in 2011 [1]. This figure represents a more than twofold rise in the last three decades and parallels the growing pandemic of obesity and the increasingly widespread adoption of Western lifestyles $[1,2]$. Worldwide prevalence is expected to continue rising in both industrialized and developing countries $[\underline{2}, \underline{3}]$, with numbers projected to reach 552 million adults by $2030[1,4]$. DM 2 is now a leading cause of death and disability and significantly increases risk for both macrovascular complications, such as atherosclerosis, and microvascular complications, such as retinitis, diabetic neuropathy, and renal disease [5]. DM2 is also strongly associated with elevated risk for other serious chronic conditions, including depression and dementia [3, 6]. Cardiovascular disease (CVD) is the primary cause of morbidity and mortality in those with DM2 [7], accounting for at least $65 \%$ of deaths in this population [ $\underline{8}$ ]. In addition, the social and economic burden of DM2 is substantial and growing rapidly. For example, DM2 accounts for at least 10 percent of all healthcare expenses in the United States, making DM2 the single most costly chronic disease [9].

\section{NIYANTRITA MADHUMEHA BHĀRATA ABHIYĀN (DIABETES FREE INDIA) STUDY}

To tackle all four dimensions of the public health challenge facing India's diabetes epidemic, a large study was conducted in 2017. Known as Niyantrita Madhumeha Bhārata Abhiyān (Diabetes Free India) and commonly abbreviated as NMB 2017, its objectives were (a) estimate the prevalence of diabetes simultaneously in all zones of India; (b) to use the pan Indian data to improve existing diabetes screening methods; (c) to conduct a cluster randomized controlled trial (RCT) to document the efficacy of a yoga-based lifestyle module for both diabetes prevention among high-risk individuals, and as an adjunct to standard treatment for bringing about normoglycemic outcome in individuals with diabetes (Nagarathna, et al., 2019; Nagendra et al., 2019).
NMB used several innovative approaches to preserve accuracy and ensure cost-effectiveness.

It employed a cluster sampling method in the screening stage, followed by selection of at risk candidates at the ward or village level for the lifestyle intervention trial. This approach enabled researchers to answer more than one research question at once. NMB also employed parallel sampling to increase sampling rate; to reduce interrater variation, the volunteers involved in data gathering underwent specialized training, with consistency checks built into various levels.

The sophisticated design of NMB 2017 allowed researchers to investigate the following:

1. What is the prevalence of diabetes in India ?

2. How to increase the accuracy of existing diabetes screening scores

3. The efficacy of Yoga to prevent diabetes

a. Does it reduce the risk of progressing from prediabetes to diabetes?

b. Does it accelerate regression to normoglycemia among people with?

Prediabetes?

c. Does it reduce the risk of progressing from high-risk to diabetes?

4. The efficacy of Yoga as a complement to standard care for individuals with diabetes:

When so implemented.

\section{YOGA AND DIABETES}

In light of the above, identifying sustainable lifestyle interventions with the potential to improve multiple factors of relevance to the management of this complex illness are of clear importance. Mind-body practices such as yoga, which capitalize on the ability of the mind to enhance physical health (and vice versa), appear particularly suited for addressing multifactorial conditions. Yoga is a traditional mind-body system originating in India over 4000 years ago [25]. In recent decades, the practice of yoga has been rising in both developed and developing countries worldwide [2629], and the field of yoga therapy is now growing rapidly [30]. The goals of yoga, a Sanskrit term meaning "yoke or union," do not center primarily on physical fitness, but rather on integration of mind, body, and spirit, cultivation of balance, calm, harmony, and awareness, and, in classic yoga traditions, the attainment of selflessness and spiritual enlightenment $[25,26]$. Of the several major branches of yoga, the most widely practiced forms include Raja (royal or 


\section{EPRA International Journal of Research and Development (IJRD) Volume: 6 | Issue: 7 | July 2021

classical) yoga and the closely related Hatha yoga, sometimes known as the yoga of activity [25, 26]. Mantra yoga, emphasizing the use of specific sounds or chants to achieve mental and spiritual transformation, was popularized in the West by Maharishi Mahesh Yogi, the founder of Transcendental Meditation (TM). Hatha and Raja yoga emphasize specific postures (asanas), including both active and relaxation or restorative poses, as well as breath control (pranayama), concentration (dharana), and meditation (dhyana), with some schools focusing primarily on restorative/meditative practices. Hatha yoga, the branch of yoga most widely practiced in the US and other Western countries, also incorporates cleansing exercises, mantras or chants, and specific hand gestures (mudras). Originally developed to prepare the body for meditation, Hatha yoga itself encompasses many different styles, including Iyengar, Ashtanga, Integral, Kundalini, Viniyoga, Vinyasa, Kripalu, and Bikram yoga $[\underline{25}, \underline{26}]$.

A growing body of evidence suggests yoga practice may reduce risk for CVD and lead to improvements in physical health and well-being in a range of populations [31-37], potentially including those with DM2.

\section{RESEARCH METHODOLOGY 1. RESEARCH DESIGN}

The present study will be a pre and post experimental study design.

\section{STUDY POPULATION}

A total of 30 employed persons with type 2 diabetes mellitus will be selected for the study on the basis of inclusion and exclusion criteria.

Space and Location:

Workplace and household area

\section{Inclusion Criteria}

Working women

Working Men

Both women and Men with type 2 diabetes mellitus

Age group: $30-55$ years.

Patients from urban areas.

\section{Exclusion Criteria}

Patients with deformities.

Other medical (except diabetes \& hypertension on regular medication) and surgical conditions (28 days after the successful completion of surgical procedure).

\section{OUTCOME MEASURES; \\ SF 36 Questionnaire}

\section{YOGA INTERVENTION PROTOCOL}

All the subjects were advised to subsist on normal diet and to continue their medication as per the prescription of their consulting physician throughout the experimentation. Reduction in their medication was made only after consultation with their physicians. Assistance of a physician was also taken for observation of patients' conditions during experimentation.

1.Laghu Shankha Prakshalana, once a week in the morning (Gherand Samhita 1/17),

2.. Agnisar, 15-30 rounds daily in the morning (Gherand Samhita 1/19).

3. Performed following practices of Pranayama-

- Sheetali Pranayama, 10-15 minutes daily in the morning (Gherand Samhita 5/74)

- Bhramari Pranayama, 10-15 minutes daily in the morning (Satyanand, 2002).

- Performed Yoga Nidra 20 minutes daily (Satyanand, 1976).

All the subjects were monitored for the following parameters before the start of experimentation (Day 1), in the end of experimentation (Day 30.)

\section{Dependent Variables}

a. Physical parameters

1. Body weight( measured by weighing machine)

2. waist circumference ( measured by measuring tape)

b. Physiological parameters

1. Serum glucose level (fasting and post prandial) ( measured by glucometer)

\section{Physical Parameters \\ Body weight}

The mean body weight of the patients was found to be $75.3( \pm 4.47) \mathrm{kg}, 73.02( \pm 4.44) \mathrm{kg}$ and $69.85( \pm 4.47) \mathrm{kg}$ on day 1, day 30 and day 60 respectively (Table 1.3). It is obvious that the mean value is lower on day 30 than that on day 1, and further lower on day 60 than that on day 1 and day 30. The values of day 1 and day 30 as well as those of day 30 and day 60 varied at 0.05 significance and those of day 1 and day 60 at 0.01 significance level. 


\section{EPRA International Journal of Research and Development (IJRD)}

In pretest and posttest, it was observed that longer duration of yogic practices was more useful in reducing the body weight.

\section{Waist circumference}

The mean waist circumference of the patients was found to be $92.25( \pm 6.29) \mathrm{cm}, 89.7( \pm 6.13) \mathrm{cm}$ and $86.25( \pm 6.29) \mathrm{cm}$ on day 1 , day 30 and day 60 respectively (Table 3.3). It is obvious that the mean value is lower on day 30 than that on day 1, and further lower on day 60 than that on day 1 and day 30. The values of day 1 and day 30 varied at non significant level, those of day 30 and day 60 also at non-significant level but those of day 1 and day 60 at 0.01 significance level.

In pretest and posttest, it was observed that longer duration of yogic practices was more useful in reducing the waist circumference.

\section{Physiological Parameters}

\section{Serum glucose level (Fasting)}

The mean serum glucose level (Fasting) of the patients was found to be $152.85( \pm 21.72) \mathrm{mg} / \mathrm{dl}, 143.3( \pm 21.56)$ $\mathrm{mg} / \mathrm{dl}$ and $134.1( \pm 19.99) \mathrm{mg} / \mathrm{dl}$ on day 1 , day 30 and day 60 respectively (Table 4.2 ). It is obvious that the mean value is lower on day 30 than that on day 1 , and further lower on day 60 than that on day 1 and day 30 . The values of day 1 and day 30 and those of day 30 and day 60 varied only non-significantly but those of day 1 and day 60 differed at 0.01 significance level.

In pretest and posttest, it was observed that longer duration of yogic practices was more useful in reducing the fasting serum glucose level.

\section{SERUM GLUCOSE LEVEL (POST PRANDIAL)}

The mean serum glucose level (Post Prandial) of the patients was found to be $201.35( \pm 34.39) \mathrm{mg} / \mathrm{dl}$, $185.75( \pm 37.23) \mathrm{mg} / \mathrm{dl}$ and $170.55( \pm 34.46) \mathrm{mg} / \mathrm{dl}$ on day 1 , day 30 and day 60 respectively (Table 5.2). It is obvious that the mean value is lower on day 30 than that on day 1 , and further lower on day 60 than that on day 1 and day 30. The values of day 1 and day 30 and those of day 30 and day 60 varied at non significant level but those of day 1 and day 60 at 0.01 significance level.

In pretest and posttest, it was observed that longer duration of yogic practices was more useful in reducing the post prandial serum glucose level.

Body weight of Diabetic Patients (Mean Value)

\begin{tabular}{|c|c|c|c|c|}
\hline Days & Mean body weight (kg) & S.D & t- value & Level of significance \\
\hline Day 1 & 75.3 & 4.47 & $2.11^{*}$ & 0.05 \\
\hline Day 30 & 73.02 & 4.44 & $2.36^{* *}$ & 0.05 \\
\hline Day 60 & 69.85 & 4.37 & $4.47^{* * *}$ & 0.01 \\
\hline
\end{tabular}

$*$ t-value of day $1 \&$ day $30, * *$ t-value of day $30 \&$ day $60, * * *$ t-value of day $1 \&$ day 60

Table ; Waist circumference of Diabetic Patients (Mean Value)

\begin{tabular}{|c|c|c|c|c|}
\hline Days & Mean waist circumference $(\mathbf{c m})$ & S.D & t- value & Level of significance \\
\hline Day 1 & 92.25 & 6.29 & $1.33^{*}$ & non significant \\
\hline Day 30 & 89.7 & 6.13 & $1.80^{* *}$ & non significant \\
\hline Day 60 & 86.25 & 6.29 & $3.09 * * *$ & 0.01 \\
\hline
\end{tabular}

$*$ t-value of day $1 \&$ day $30, * *$ t-value of day $30 \&$ day $60, * * *$ t-value of day $1 \&$ day 60

Table ; Serum glucose level (Fasting) of Diabetic Patients (Mean Value)

\begin{tabular}{|c|c|c|c|c|}
\hline Days & Mean serum glucose level $(\mathbf{m g} / \mathbf{d l})$ & S.D & t- value & Level of significance \\
\hline Day 1 & 152.85 & 21.72 & $1.43^{*}$ & non significant \\
\hline Day 30 & 143.3 & 21.56 & $1.43^{* *}$ & non significant \\
\hline Day 60 & 134.1 & 19.99 & $2.91 * * *$ & 0.01 \\
\hline
\end{tabular}

$*$ t-value of day $1 \&$ day $30, * *$ t-value of day $30 \&$ day $60, * * *$ t-value of day $1 \&$ day 60 


\section{EPRA International Journal of Research and Development (IJRD) Volume: 6 | Issue: 7 | July 2021 - Peer Reviewed Journal}

Table ; Serum glucose level (Post Prandial) of Diabetic Patients (Mean Value)

\begin{tabular}{|c|c|c|c|c|}
\hline Days & Mean serum glucose level (mg/dl) & S.D & t- value & Level of significance \\
\hline Day 1 & 201.35 & 34.39 & $1.41^{*}$ & non significant \\
\hline Day 30 & 185.75 & 37.23 & $1.37^{* *}$ & non significant \\
\hline Day 60 & 170.55 & 34.46 & $2.89^{* * *}$ & 0.01 \\
\hline
\end{tabular}

$* \mathrm{t}$-value of day $1 \&$ day $30, * * \mathrm{t}$-value of day $30 \&$ day $60, * * * \mathrm{t}$-value of day $1 \&$ day 60

\section{DISCUSSION}

The body weight of all diabetic population before and after yoga intervention were assessed in this study on day 1,30 and 60 respectively.

The waist circumference of all diabetic population before and after yoga intervention were assessed in this study on day 1,30 and 60 respectively.

The fasting and post prandial serum glucose level of all diabetic population before and after yoga intervention were assessed in this study on day 1,30 and 60 respectively.

After comparing the scores of body weight between the pretest and posttest working population the difference in the score was found to be statistically highly significant. The mean scores of the posttest were higher than the pre intervention. Therefore it was indicated that the working population with inclusion of yoga was feeling much better than before.

After comparing the scores of waist circumference between the pretest and posttest working population, the difference in the score was found to be statistically highly significant. The mean scores of the posttest were higher than the pre intervention. Therefore it was indicated that working population with inclusion of yoga was feeling much better than before.

After comparing the scores of Serum glucose level fasting and post prandial between the pretest and posttest working population, the difference in the score was found to be statistically highly significant. The mean scores of the posttest were higher than the pre intervention. Therefore it was indicated that the of the working population with inclusion of yoga was feeling better than before.

\section{CONCLUSION}

The results indicated that the working population(30-55) with type 2 diabetes mellitus was in better physical state with inclusion of Yoga in there daily routine.

\section{REFRENCES}

1. Lam D. W., LeRoith D. The worldwide diabetes epidemic. Current Opinion in Endocrinology, Diabetes and Obesity. 2012;19(2):93-96. doi: 10.1097/med.0b013e328350583a. [ [PubMed] [CrossRef] [Google Scholar]

2. Chen L., Magliano D. J., Zimmet P. Z. The worldwide epidemiology of type 2 diabetes mellitus-present and future perspectives. Nature Reviews Endocrinology. 2012;8(4):228-236. doi: 10.1038/nrendo.2011.183. [PubMed] [CrossRef] [Google Scholar]

3. Inzucchi S. E., Bergenstal R. M., Buse J. B., et al. Management of hyperglycemia in type 2 diabetes: a patient-centered approach: position statement of the American Diabetes Association (ADA) and the European Association for the Study of Diabetes (EASD) Diabetes care. 2012;35(6):1364-1379. doi: 10.2337/dc12-0413. [PMC free article] [PubMed] [CrossRef] [Google Scholar]

4. International Diabetes Federation. IDF Diabetes Atlas. Brussels, Belgium: International Diabetes Federation; 2011. [Google Scholar]

5. Fowler M. J. Microvascular and macrovascular complications of diabetes. Clinical Diabetes. 2011;29(3):116-122. doi: 10.2337/diaclin.29.3.116. [CrossRef] [Google Scholar]

6. American Diabetes Association. Standards of medical care in diabetes-2014. Diabetes Care. 2014;37(supplement 1):S14-S80. doi: 10.2337/dc14-s014. [ PubMed] [CrossRef] [Google Scholar]

7. Joseph J. J., Golden S. H. Type 2 diabetes and cardiovascular disease: what next? Current Opinion in Endocrinology, Diabetes and Obesity. 2014;21(2):109-120. doi: 10.1097/med.0000000000000044. [PMC free article] [PubMed] [CrossRef] [Google Scholar]

8. American Heart Association. Cardiovascular Disease \& Diabetes, 2012, http://www.heart.org/HEARTORG/Conditions/Diab etes/WhyDiabetesMatters/Cardiovascular-DiseaseDiabetes_UCM_313865_Article.jsp/

9. Petersen M. Economic costs of diabetes in the US in 2012. Diabetes Care. 2012;36(4):1033-1046. 
doi: 10.2337/dc12-2625. [PMC free article] [ PubMed] [CrossRef] [Google Scholar]

10. Innes K. E., Vincent H. K. The influence of yogabased programs on risk profiles in adults with type 2 diabetes mellitus: a systematic review. Evidencebased Complementary and Alternative Medicine. 2007;4(4):469-486. doi: 10.1093/ecam/nello3. [PMC free article] [PubMed] [CrossRef] [ Google $\underline{\text { Scholar] }}$ 